\title{
Primer hallazgo de Henricia obesa (Sladen, 1889) (Asteroidea: Echinasteridae) en el mar peruano
}

\section{First records of Henricia obesa (Sladen, 1889) (Asteroidea: Echinasteridae) in the Peruvian sea}

\section{Carlos Paredes ${ }^{1}$ y Alex Gamarra ${ }^{2}$}

\begin{abstract}
${ }^{1}$ Laboratorio de Biología y Sistemática de Invertebrados Marinos, Facultad de Ciencias Biológicas, UNMSM, Apdo. 11-0058, Lima 11, Perú. E-mail:

cparedesq@unmsm.edu.pe

2 Laboratorio de Ecología, Facultad de Ciencias Biológicas, UNMSM, Apdo. 110058, Lima 11, Perú.
\end{abstract}

Presentado: 11/04/2006 Aceptado: 18/07/2006

\section{Resumen}

Se reporta por primera vez para el Perú a la «estrella de mar» Henricia obesa (Sladen, 1889) (Asteroidea: Echinasteridae), hallada en aguas frías, dentro de los límites de la Provincia Peruana.

Palabras clave: Asteroidea, Echinasteridae, Henricia obesa, nuevo registro, Perú Abstract

The star sea Henricia obesa (Sladen, 1889) (Asteroidea: Echinasteridae) is reported for the first time from Peruvian sea.

Key words: Asteroidea, Echinasteridae, Henricia obesa, new records, Perú
D esde el trabajo clásico de Clark (1910) los Echinodermata del mar peruano han sido uno de los grupos de invertebrados marinos muy poco estudiados, no obstante su importancia ecológica en los diferentes ambientes marinos en los que suelen ser los depredadores de alto nivel. Madsen (1956) realizó un estudio zoogeográfico considerando la costa peruana desde Punta Aguja (60 S), toda la costa chilena, y la costa sur de Argentina, hasta los $42^{\circ} \mathrm{S}$, llegando a registrar 37 especies de Asteroidea, donde estaban incluidas H enricia obesa (Sladen, 1889) en el sur de Chiley Argentina, H. Studeri (E. Perrier, 1891), en el Cabo de Hornosy $H$ enricia sp. en la Provincia Peruana, sin datos de colecta. Recientemente Hooker et al.,(2005) registraron 7 especies de Asteroidea, incluyendo dos hallazgos nuevos, en los límites de la Isla Lobos de Tierra (06 $55^{\prime} 5^{\prime \prime}$ S), dentro del ámbito de la Provincia Peruana. Si consideramos las otras cuatro especies conocidas de las aguas frías de la Corriente Peruana ( L uidia magellanica, Patiria chilensis, Stichaster striatus y Meyenaster gelatinosus), son once los asteroideos, que han sido reportados hasta el momento en las aguas someras del mar peruano, al sur de Punta Aguja ( 6 o S). Este trabajo informa sobre el primer registro de $\mathrm{H}$ enricia obesa (Sladen, 1889)(A steroidea: Echinasteridae) en aguas dela Provincia Peruana.

Las muestras fueron tomadas en el sublitoral de la Bahía de Matarani Arequipa , Perú (165ํ'S - 720ㅜ'W) (Figura 1) mediante buceo semi-autónomo. La profundidad de la colecta fue de $28 \mathrm{~m}$. Los 3 ejemplares de «estrellas de mar» se fijaron con formol al 7\% neutralizado con bórax y conservaron en alcohol etílico al 70\%. La determinación taxonómica se realizó en el laboratorio utilizando el trabajo de Bernasconi (1980). Se hizo mediciones y se tomó fotografías. El material ha sido incorporado a la colección del Laboratorio de Biologíay Sistemática de Invertebrados Marinos de la Facultad de Ciencias Biológicas de la Universidad Nacional Mayor de San Marcos.

Se registra por primera vez la «estrella de mar» $\mathrm{H}$ enricia obesa (Sladen, 1889) en el mar peruano, encontrada en el Puerto de Matarani, D epartamento de A requipa.

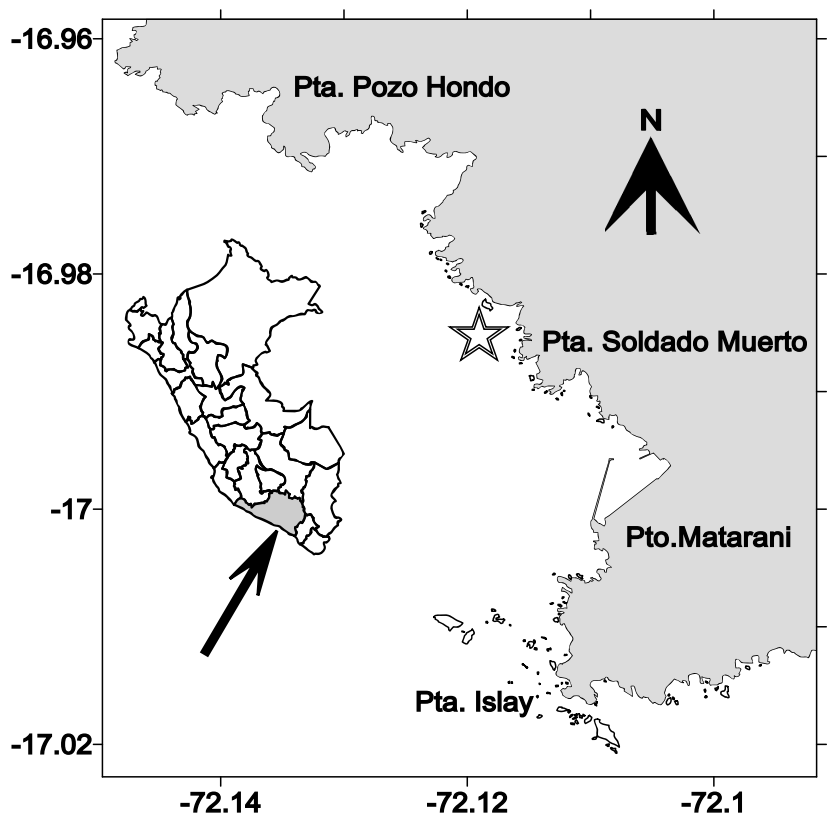

Figura 1. Mapa de la zona costera de la provincia de Islay. La estrella indica el lugar de colecta de Henricia obesa (Salden, 1889).

\section{Familia Echinasteridae}

\section{Genero Henricia Gray 1840}

\section{Henricia obesa (Sladen 1889)}

\section{(Figura 2)}

Cribrella obesa Sladen 1889:544, pl.96, fig. 3, 4; 98, fig. 5,6. Cribrella hyadesi, Perrier, 1891:100.

Henricia pagenstecheri, Koeler, 1923: 60.

Henricia obesa, Fisher, 1940: 164; Mortensen, 1941:3; Madsen, 1965:11; Bernasconi, 1966: 169; Bernasconi, 1973:308;

Bernasconi, 1980: 250-252.

Descripción: En general, las características de nuestro material coinciden con la descripción de Bemasconi (1980). Los cinco brazos son largos, están inflados en su tercio proximal, y for- 
man profundas hendiduras en el borde del pequeño disco. La coloración es naranja intenso, algo más claraen lasuperficieactinal.

Distribución: Parte sur de América del Sur, desdeCabo de Hornos, con dirección norte, hasta G olfo de San Jorge por el lado de Argentina, y en el lado chileno hasta el extremo norte de Chiloé (Madsen, 1956, Bernasconi, 1980). También ha sido reportada en Iquique, Chile (Meissner, 1896). Según Fisher, 1940) es una especie típica de la Meseta de las Islas Malvinas, y Mortensen (1941) la cita para Tristan da Cunha. Además, el primer reporte de esta especie, fuera de la región MagallánicaIslas Malvinas, fue realizado en la Bahía de Lusitania (Nueva Zelanda) (Clark, 1962).

Hábitat: En fondos rocosos con arenay conchuela.

Localidad: Bahía Matarani (170ㅜㅇㅡ, $\left.72^{\circ} 06^{\prime} \mathrm{W}\right)$, Arequipa,frente a Punta Soldado Muerto, Perú, entre 25 y 30 m de profundidad. Sin embargo, tiene un rango batimétrico entre 0y 242 m (Bernasconi, 1980).

Material examinado.- 1 lote, 3 ejemplares: Diámetro del ejemplar más grande, 91,5 mm; Col. A. Gamarra; LaBSIM.

Comentario.- Si se tiene en cuenta que los muestreos realizados anteriormente fueron hechos en profundidades $<20 \mathrm{~m}$, éste límite batimétrico en los trabajos, podría ser la causa del no hallazgo anterior de esta especie. Sin embargo, la temperatura también puede ser considerada, pues, siendo $\mathrm{H}$. obesa propia de aguas subantárticas (Bernasconi, 1980), su desplazamiento hasta la Bahía de Matarani se explicaría por el periodo decadal frío iniciado en 1997 (Chavez, en prensa).

Con el registro de $\mathrm{H}$ enricia obesa (Sladen, 1889) se incrementa a 12 el número de asteroideos hallados en el mar peruano al sur de Punta Aguja.

Porotra parte, Larraín (1995) lista 5 especies del genero H enriaa para Chile, con algunas imprecisiones sobre sinonimia. Por esta razón hemos preferido mantener lo indicado por Bernasconi(1980).

\section{Agradecimientos}

Al profesor $O$ swaldo Cornejo, por las facilidades brindadas en el Laboratorio de E cología de la Universidad Nacional Mayor de San Marcos. Al Terminal internacional del Sur S.A., Consultora G older Associates Perú S.A. De consideración especial al MSc. Rene Lozada y al personal que participó en la Caracterización del Ecosistema Marino en Bahía Matarani, Arequipa, Perú.

\section{Literatura citada}

Bernasconi, I. 1980. Asteroideos Argentinos VII. Familia Echinasteridae. Rev. Museo Argentino de Ciencias Naturales «Bernardino Rivadavia». 5(12): 247-258, 3 pls.

Chavez, F.P. (in press). Biological consequences of interanual to multidecadal variability. Chapter 17. In Volume 13 of The Sea. A. Robinson and K. Brink (eds.).

Clark, H.L. 1910. The Echinoderms of Peru. Bull. Mus. Comp. Zoology. 52(17): 321-358.

Clark, A.M. 1962. Asteroidea.B.A.N.Z.A.R. Exped. Rep. (B)9:1104.

Fisher, W.K. 1940. Asteroidea. Discovery Reports XX:69-306, pls. 1-23.

Hooker, Y.,F. Solís y M. Llellish. 2005. Equinodermos de las Islas Lobos de Afuera (Lambayeque, Perú). Rev. peru. biol. 12(1):77-82.

Larrain, A.P. 1995. Biodiversidad de Equinodermos chilenos: Estado actual del conocimiento y sinopsis biosistemática. Gayana Zool. 59(1):73-96.

Madsen, F. J. 1956. Asteroidea (With a survey of the Asteroidea of the chilean Shelf). Repts. Lund Univ. Chile Exped. (19481949), 24:1-53.

Meissner, M. 1896. Die von Herrn Plate aus Chile und Feuerland heimgebrachten seesterne. Archiv fur Naturgeschichte. 62(1): 101.

Mortensen, Th. 1941. Echinoderms of Tristan da Cunha. Res. Norw. Sc. Exp. To Tristan da Cunha, 1937-38 (7):3, pl. 1, fig. 2.

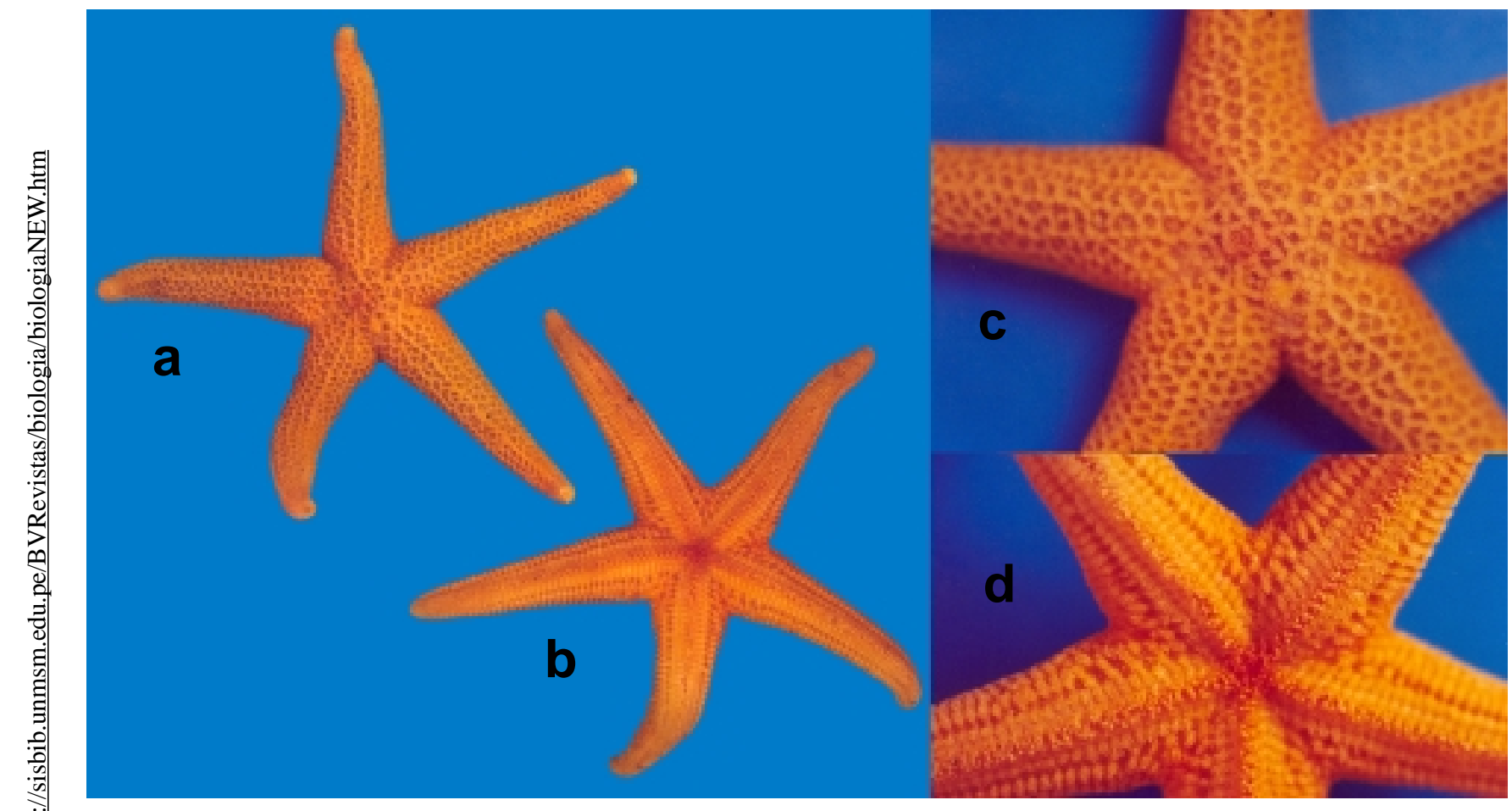

Figura 2. Henricia obesa: (a) Vista aboral, (b) Vista oral, (c) Detalle del disco en vista aboral, (d) Detalle del disco en vista oral. Diámetro 91, $5 \mathrm{~mm}$. 